\title{
Defect Range and Evolution in Swift Xe-Ion Irradiated Pure Silver Studied by Positron Annihilation Technique
}

\author{
J. DryzeK ${ }^{a, *}$, P. HorodeK ${ }^{a, b}$ AND V.A. SKuratov ${ }^{b, c, d}$ \\ ${ }^{a}$ Institute of Nuclear Physics, Polish Academy of Sciences, PL-31342 Kraków, Poland \\ ${ }^{b}$ Joint Institute for Nuclear Research, Joliot-Curie 6, 141980 Dubna, Moscow region, Russia \\ ${ }^{c}$ National Research Nuclear University MEPhI, Kashirskoye sh. 31, 115409 Moscow, Russia \\ ${ }^{d}$ Dubna State University, Universitetskaya 19, 141980 Dubna, Moscow region, Russia
}

\begin{abstract}
Variable energy positron beam and positron lifetime spectroscopy were used to study pure silver samples exposed to irradiation with swift $\mathrm{Xe}^{26+}$ ions of energy $167 \mathrm{MeV}$ with different dose: of $10^{13}, 5 \times 10^{13}$ and $10^{14}$ ions $/ \mathrm{cm}^{2}$. The positron lifetime spectroscopy revealed the presence of dislocations or vacancies associated with dislocations. They are distributed at the depth of about $6 \mu \mathrm{m}$, and this correlates with the ion implantation range, i.e. $9 \mu \mathrm{m}$. However, some defects are observed also to a depth of about $18 \mu \mathrm{m}$. At the depth less than $1 \mu \mathrm{m}$ from the entrance surface strong dependence of positron diffusion length on the dose is observed. It indicates the presence of interstitial atoms and/or dislocation loops as a result of $\mathrm{Xe}^{26+}$ ions implantation.
\end{abstract}

DOI: $10.12693 /$ APhysPolA.132.1585

PACS/topics: $61.80 . \mathrm{Jh}, 61.82 . \mathrm{Bg}, 78.70 . \mathrm{Bj}$

\section{Introduction}

Studies of radiation damage are important for evaluation of a tolerance of materials to extreme radiation conditions [1]. That is important for the nuclear and space technology where materials and devices are exposed significantly to an impact of energetic particles [2,3]. Additionally, the implantation of energetic particles including ions have been used for doping of semiconductors and other materials to create desired properties [4].

Swift heavy ions implanted into solids lose their energy by electronic excitations and nuclear collisions. It was established that electronic excitations play an important role in damage processes of metallic solids [5]. Interaction time of ion with target atom is estimated for about $10^{-18} \mathrm{~s}$. During this time the emission of delta electrons occurs, and a cylindrical track core with positive charge forms along the ion path. The energy of delta electrons is less than tens of $\mathrm{keV}$ and they cannot involve the displacement of atoms via direct collisions. Recombination and thermalization of theses electrons follow by phonon interaction. The release of this energy to the lattice in such a case takes much longer, i.e., about $10^{-13}-10^{-10} \mathrm{~s}$. This causes the rapid increase of the local temperature and creation of thermal spikes along the ion path [6]. If temperature increases above the melting point of the solid then the molten phase occurs. Rapid cooling of this region results in freezing crystalline defects including open volume defects like vacancies, their clusters and stacking fault tetrahedra.

In turn, the collision of ion with target nucleus induces directly an atom and atoms displacement and creation of displacement cascades. They are also the source of point

*corresponding author; e-mail: jerzy.dryzek@ifj.edu.pl defects and stacking fault tetrahedra localized in a cylinder centered around the ion trajectory path. The point defects will evolve in time, vacancy and interstitial atoms can recombine or annihilate in sinks or agglomerate generating vacancy clusters and/or interstitial clusters. The cascades can collapse to dislocation loops, too. Also interstitial atoms and interstitial clusters, like a crowdion can develop in dislocation loops. The temperature, dose and type of crystalline structure of a target are important factors of behavior of defects and their final state.

It the literature, a large difference between ion irradiation damage depth and the actual ion ranges was reported. The experiments performed for $\mathrm{Cu}, \mathrm{Mo}, \mathrm{Pt}$ and $\alpha$-Fe have shown the change of material properties far beyond the ion range, simulated e.g. using SRIM code $[7,8]$. This phenomena is called "long range effect" (LRE) because, as reported by some of the authors a change in the dislocation structure occurs at distances of tens of $\mu \mathrm{m}$ even when the predicted ion range was only a few hundred nm. Typically, it is explained by the stressinduced dislocation structure formation during the implantation [9]. However, studies of $\mathrm{Lu}$ et al. who used $3 \mathrm{MeV} \mathrm{Au}$ ions for irradiation of single crystalline $\mathrm{Ni}$, $\mathrm{NiCo}$, and $\mathrm{NiFe}$ alloys did not confirmed LRE for the alloys [10]. Nevertheless, in case of Ni they observed the damage range at the depth of $500 \mathrm{~nm}$ whereas the ion range was about $300 \mathrm{~nm}$. The authors explained this discrepancy by the higher diffusivity of defects in Ni than in its alloys. It can be enhanced by a higher stress field gradient after irradiation by $\mathrm{Au}$ ions. One should point out that observation of defects is not a simple task. One cannot exclude generation of additional defects during preparation for microscopic observation e.g. TEM which can overlap the defects induced by ion implantation. The preparation of the sample before implantation is also important, because residual defects can camouflage the new defects. We argue that positron annihilation technique 
allows us to omit such issues. Additionally its selectivity and sensitivity to open volume defects is extremely high. No special preparation of samples after irradiation is needed.

So far the slow positron beam technique was applied to detection of defect range in implanted samples [11, 12]. Mazzoldi et al. studied silica glass implanted by $\mathrm{Ar}^{+}$ions of energy $30 \mathrm{keV}$ using this technique [11]. They found defect distribution extending more than twice deeper than ion range. However, the analysis of positron data in such a case is complicated because the positron diffusion process can mask the defect distribution induced by implantation. Additionally, silicon glass is not a perfect structure itself, it contains defects and imperfection which can affect the results. In the presented paper we intend to applied much simpler experimental method free of these drawbacks.

The aim of the paper is to detect the defect depth profile in the $\mathrm{Xe}^{26+}$ ion range and beyond of it. The ions were implanted into silver samples with different dose. We intend to recognize the type of defects created in sliver exposed to the implantation of the heavy ions with the energy of $167 \mathrm{MeV}$ and search for LRE. For this purpose the variable energy slow positron beam and conventional positron lifetime spectroscopy will be used.

\section{Experimental details}

\subsection{Sample preparation}

Several silver plates purchased from Nilaco with dimensions $1 \times 1 \times 0.1 \mathrm{~cm}^{3}$ were firstly annealed at $400^{\circ} \mathrm{C}$ for $1 \mathrm{~h}$ in the $\mathrm{N}_{2}$ flow atmosphere and slowly cooled down to the room temperature. Then all of them were chemically etched to remove possible chemical products from the surface using 1:3 nitric acid and distilled water. For such prepared samples only a single-lifetime component equal to $155 \pm 1$ ps in the measured LT spectra was resolved. The obtained value is higher than the value for the bulk in pure silver equal to 130 ps [13], it indicates the presence of residual defects like dislocation in the samples. Despite this we decided to perform implantation in order to observe also the overlapping of these defects with those introduced by implantation of the $\mathrm{Xe}^{26+}$ ions.

The implantation of four samples was performed at IC100 cyclotron at Flerov Laboratory of Nuclear Reactions at Joint Institute for Nuclear Research (JINR) in Dubna, Russia. $\mathrm{Xe}^{26+}$ heavy ions with energy of $167 \mathrm{MeV}$ and different doses, namely $10^{13}, 5 \times 10^{13}$ and $10^{14}$ ions $/ \mathrm{cm}^{2}$ were applied. $\mathrm{Xe}^{26+}$ belongs to highly energetic heavy ion similar to fission fragments. The average ion flux was $5 \times 10^{9} \mathrm{~cm}^{-2} \mathrm{~s}^{-1}$. Temperature during irradiation did not exceed $80^{\circ} \mathrm{C}$.

\subsection{Positron annihilation measurements}

VEP measurements were performed also at JINR Dubna. The monoenergetic positron beam with intensity of $c a .10^{5} \mathrm{e}^{+} / \mathrm{s}$ and diameter of $5 \mathrm{~mm}$ was used. The energy of injected positron was ranged from 0.5 to $35 \mathrm{keV}$. The Doppler broadening spectrometer composing HPGe detector with energy resolution of $1.20 \mathrm{keV}$ at
$511 \mathrm{keV}$ registered annihilation photons resulting from positron annihilation in a sample. The annihilation line was analyzed by extraction of the so-called $S$ parameter value which is given as a ratio of area under the central part of the annihilation line to the total area under this line. It defines the contribution of annihilating electronpositrons pairs with low momentum present which occur mostly at open volume defects.

The PALS measurements was performed at the fastfast spectrometer based on $\mathrm{BaF}_{2}$ scintillators with timing resolution of about $250 \mathrm{ps}$. The isotope ${ }^{22} \mathrm{Na}$ with activity $32 \mu \mathrm{Ci}$ enveloped into a $7 \mu \mathrm{m}$ thick kapton foil was placed between two samples, the ion beam entrance surface placed towards the source. The sandwich was located in front of the scintillator detectors of the spectrometer. The analysis of obtained spectra including $10^{6}$ counts was done by LT program [14]. Background, and source correction were taken into account.

\section{Simulation of Xe ion distribution}

In Fig. 1a SRIM calculations [15] of ion ranges and created vacancy distributions for the case of $167 \mathrm{MeV} \mathrm{Xe}^{26+}$ ion implantation into silver are shown, $10^{4}$ ions trajectories were taken into account. The projected range, end of the peak is located at the depth of $8.2 \mu \mathrm{m}$ with struggling of $0.5 \mu \mathrm{m}$. The $\mathrm{Xe}^{26+}$ concentration as well as vacancy density arises proportionally according to these simulations. One should emphasize the increase of the vacancy amount by the factor about ten at the end of ion range in comparison to the vicinity of the entrance surface. This could be detected by positron measurements.

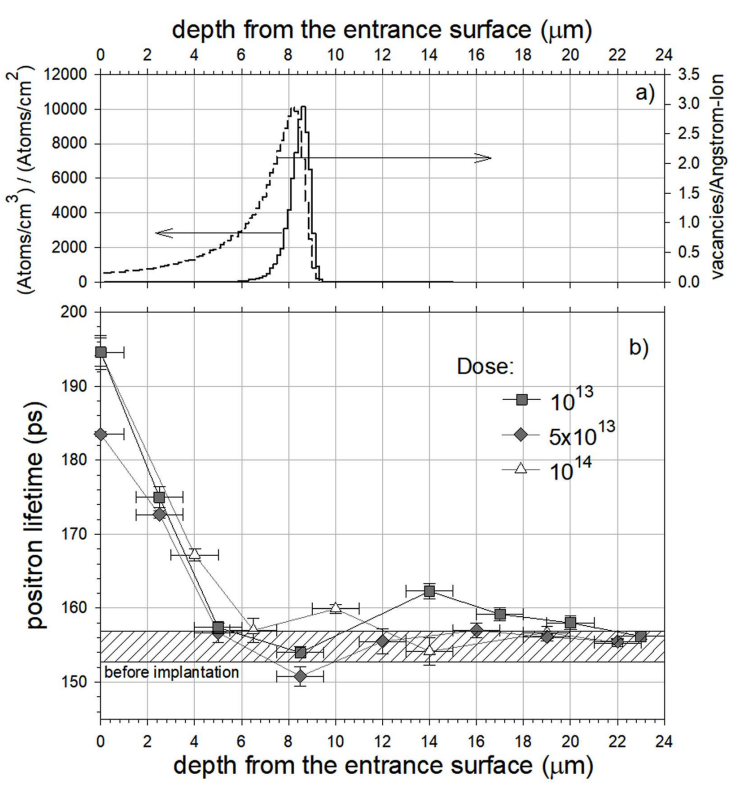

Fig. 1. SRIM calculations of ion (a) (left axis) and vacancy concentration (right axis) as a function of depth after $\mathrm{Xe}^{26+}$ ions implantation with energy of $167 \mathrm{MeV}$ in pure Ag. The depth distribution of positron lifetime component obtained in the etching experiment for $\mathrm{Ag}$ implanted with different dose (b). The hatched region represents the value of positron lifetime resolved for $\mathrm{Ag}$ samples before implantation. 


\section{Results and analysis of the measurements}

\subsection{VEP results}

The measured values of $S$ parameter as a function of the positron incident energy are depicted in Fig. 2. The closed circles represent values obtained for the reference not implanted silver sample and other symbols for implanted samples with different dose. The obtained de-

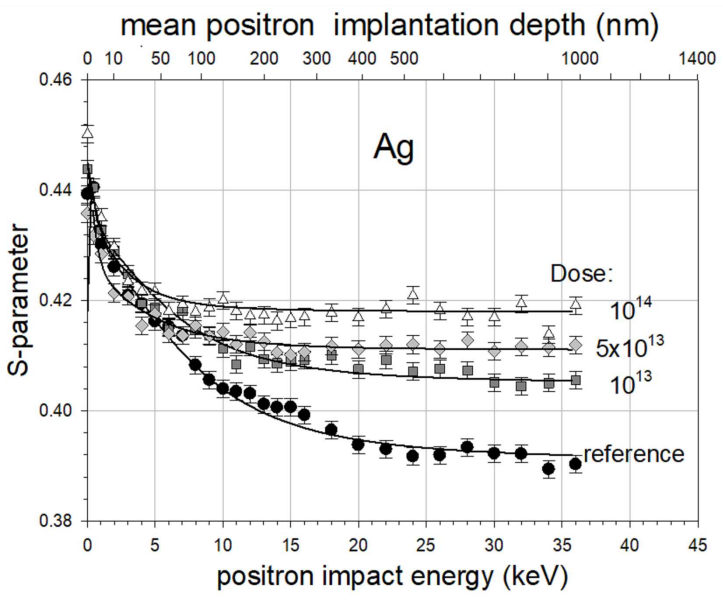

Fig. 2. The measured annihilation line shape parameter as a function of the positron incident energy for $\mathrm{Ag}$ samples irradiated by $\mathrm{Xe}^{26+}$ ions with different dose. The top axis represents the mean positron implantation depth, Eq. (4) calculated as follows: $\bar{x}[\mathrm{~nm}]=3.48 \times E^{1.576}$, where $E$ is the positron impact energy in $\mathrm{keV}$. The solid lines represent the best fit of Eq. (A1) to the corresponding experimental points. The values of adjustable parameters are gathered in Table I.

\section{TABLE I}

The values of the adjustable parameters determined in the fitting procedure of Eq. (A1) to the experimental points in Fig. 2.

\begin{tabular}{c|c|c|c}
\hline \hline $\begin{array}{c}\text { Dose } \\
{\left[\text { ions } / \mathrm{cm}^{2}\right]}\end{array}$ & $\begin{array}{c}L_{+} \\
{[\mathrm{nm}]}\end{array}$ & $S_{\text {surf }}$ & $S_{\text {int }}$ \\
\hline $10^{13}$ & $59(7)$ & $0.431(8)$ & $0.405(2)$ \\
$5 \times 10^{13}$ & $31(6)$ & $0.425(8)$ & $0.411(1)$ \\
$10^{14}$ & $24(7)$ & $0.431(13)$ & $0.417(2)$ \\
$\mathrm{Ag}$, reference & $97(10)$ & $0.431(8)$ & $0.391(4)$
\end{tabular}

pendences are typical for many metals, i.e., the decrease of the $S$-parameter value with the increase of the positron incident energy. The saturation value of the $S$-parameter at higher energies increases with the increase of the dose indicating the presence of defects which traps positrons. For description of the obtained dependences one can use the results of the diffusion trapping model presented in Appendix. (In our consideration we neglected ephithermal positrons trapped at the surface.) The solid lines in Fig. 1 represent the best fit of Eq. (A1) to the experimental points for the samples. Three adjustable parameters were used in the procedure, their values are gathered in
Table I. The positron diffusion length value is highest for the reference sample, i.e., about $97(10) \mathrm{nm}$. Such a value is typical for metals, however, implantation of $\mathrm{Xe}^{26+}$ ions gradually reduces this value to $24(7) \mathrm{nm}$ for the highest dose, i.e., $10^{14}$ ions $/ \mathrm{cm}^{2}$. Following this the value of the $S$-parameter in the interior, i.e., $S_{\text {int }}$ of the samples gradually increases. This is explained by the fact that the implantation introduces defects, including open volume defect which trap positrons and terminate their diffusion process as well. The value of the $S$-parameter at the surface does not alter in comparison to the reference sample, it indicates the lack of creation surface defects during implantation. The obtained results are valid at the depth of about $1 \mu \mathrm{m}$ from the entrance surface, Fig. 1, top axis. At this depth the defects distribution does not change with the depth increase. In other ways much complex relation than Eq. (A1) should be used for data description. The obtained dependences are very similar to those obtained for samples of pure Fe irradiated by $\mathrm{Xe}^{26+}$ ions [16].

\subsection{Positron lifetime depth distribution results}

VEP experiment is suitable for scanning the depth of about $1 \mu \mathrm{m}$ from the entrance surface. According to the simulations, Fig. 1a, the region of damage caused by implantation is extended up to the depth of $9.5 \mu \mathrm{m}$, thus it is much deeper range. For scanning such a large depth, we proposed another technique, which was used with success for studies of a subsurface zone created after dry sliding, blasting or machining. In that case the measurement of the positron lifetime spectrum was performed but after etching off some layer from the damage sample (see e.g., Ref. [17]). The sequenced etching allows us to determine the depth profile of the positron lifetime spectrum. For our implanted samples the thickness of the etched layer must be much less than $9.5 \mu \mathrm{m}$. We chosen the thickness of about $2 \mu \mathrm{m}$. In all spectra only single lifetime component was resolved with $\chi^{2}$ close to unity. In Fig. 1b we depicted the obtained dependence of the positron lifetime value as the function of the thickness of the etched layer or depth from the entrance surface. It can be noticed that this value decreases from about 190-200 ps with the depth increases to the value obtained before implantation, i.e. $155 \mathrm{ps}$, tagged by the hatched rectangular area. This is at the depth of about $5-6 \mu \mathrm{m}$, and it only slightly less that value for the ion range, i.e., $9.5 \mu \mathrm{m}$, Fig. 1a. This can be caused by the effect of overlapping the introduced defects by the residual defects. The positron lifetime values lower than value for positron lifetime in a monovacancy for the perfect lattice, i.e. 208 ps [13], indicate the presence of dislocations or vacancies located near or at dislocation lines. This differs from iron also irradiated by $\mathrm{Xe}^{26+}$ ions, where vacancy clusters were observed [16]. At this stage we quit more sophisticated analysis of the measured spectra, e.g. taking into account the positron implantation profile because the satisfactory description with single lifetime component only. Adding more components could not improve the $\chi^{2}$ value, and can lead to the misleading conclusions. 
In comparison to the results from VEP the obtained dependences are hardly affected by the implanted dose. This can be explained by the fact that positron diffusion length depends (see Eq. (A1)) not only on the positron trapping rate, i.e., the defect concentration but also on the positron diffusion coefficient. Diffusion can be limited by the presence of other defects, like interstitial atoms, dislocations or fault tetrahedra, which can scatter randomly walk positrons reducing the diffusion coefficient. Positron lifetime spectra is sensitive only to open volume defects which additionally can localize positrons.

The positron lifetime for all dependences decreases linearly with the depth increase and at the depth of $10 \mu \mathrm{m}$ the value of about 155 ps is reached, Fig. 1b. Deeper this value does not change, significantly. One should keep in mind that the implantation depth of ${ }^{22} \mathrm{Na}$ positrons is about $18 \mu \mathrm{m}$ in silver, thus about $42 \%$ of positrons only annihilate in the layer $6-7 \mu \mathrm{m}$ thick from the entrance surface. As it was discussed in Ref. [18], the measured linear decrease of the mean positron lifetime corresponds also to the linear decrease of the local mean positron lifetime, which reflects the local properties. Then we argue that amount of defects introduced by implantation almost linearly decreases with the depth increase as well. This is in contradiction to the results presented in Fig. 1a, where the vacancy concentration increases with the depth increase and at the end of the ion range the maximum is reached. The explanation of the discrepancy can be as follows. The SRIM/TRIM simulation does not take into account the evolution of created defects, their diffusion, annihilation and reactions between them. But such process must occur if the implantation takes place in the room or higher temperature. This can modified the initial defect distribution, and change their types as well.

It is worth noticing that at the depth of above $10 \mu \mathrm{m}$ for two samples with dose of $10^{14}$ and $10^{13}$ ions $/ \mathrm{cm}^{2}$ slight increase of about a few ps of the positron lifetime value is observed. However, it decreases at the depth of $20 \mu \mathrm{m}$ to the value $c a .155 \mathrm{ps}$ resolved for the reference sample. Such an increase beyond the ion range was observed also for the sample of $\mathrm{Fe}$ [16] and $\mathrm{Cu}$ [19] irradiated by $\mathrm{Xe}^{26+}$. In these cases the increase of the positron lifetime was in the range between 5 to 10 ps above the bulk lifetime. This can support the creation of some defects beyond the ion range in $\mathrm{Ag}$ as well. It is interesting that this effect generates defects additional to the residual defects present in the implanted samples. However, this effect is extended only at the depth of lower than twice the ion range. Certainly, such an effect is not predicted by SRIM/TRIM simulations, Fig. 1a. From the results we are not able to distinguish the kind of defects and their amount at this range. To obtain such an information more sensitive technique must be developed.

We should state as follows: the residual defects beyond ion implantation range are not able to mask the additional defects in this case. We argue that the origin of these defects can be connected to their diffusion from the region closer to the surface, or stress-induced dislocation motion caused by swelling of sample after ions implantation. We would not argue that the presented effect is the LER reported by Sharkeev et al. [7]. It seems it can be treated as the hallo of defects around the ion paths. However, it is very small effect. At this stage we are not able to point out what kind of defects are present in the hallo region. To obtain such an information more sensitive technique must be develop focusing at the depth beyond the ion range.

\section{Conclusions}

The implantation of swift $\mathrm{Xe}^{26+}$ ions with energy of $167 \mathrm{MeV}$ in $\mathrm{Ag}$ samples with some residual defects revealed the induced defect profile. The range of the defects is about $5-6 \mu \mathrm{m}$ and is hardly affected by the dose. This depth corresponds to the depth of ion range calculated using SRIM/TRIM code, which is about $9 \mu \mathrm{m}$. The small discrepancy can result from the fact that Ag sample contained some residual defects, which can mask the induced defects. For one of the samples we observed the small increases of the positron lifetime which can be interpreted as the presence of defects beyond the ion range. The significant increase of defect concentration at the depth of about $1 \mu \mathrm{m}$ was revealed using VEP beam. In that case the concentration significantly depends on dose of implanted ions. This can result from presence of interstitial atoms and other defects which only scatter randomly walking positrons.

\section{Appendix}

According the diffusion trapping model the measured $S$ parameter values versus the positron incident energy $E$ can be expressed as follows (see Eq. (8) in Ref. [20]):

$$
\begin{aligned}
& S(E)=S_{\text {int }}+ \\
& \quad\left(S_{\text {surf }}-S_{\text {int }}\right) \int_{0}^{\infty} \mathrm{d} x p(x, E) \exp \left(-\frac{x}{L_{+}}\right),
\end{aligned}
$$

where $S_{\text {int }}=\frac{S_{\text {bulk }}+S_{\text {def }} k_{0} / \lambda_{\text {bulk }}}{1+k_{0} / \lambda_{\text {bulk }}}, S_{\text {surf }}, \quad S_{\text {bulk }}$, and $S_{\text {def }}$ represent values attributed to the annihilation at the surface, in bulk and at a defect, respectively. $L_{+}$is a positron diffusion length equal to $L_{+}=$ $\sqrt{D_{+} / \lambda_{\text {bulk }}} / \sqrt{1+k_{0} / \lambda_{\text {bulk }}}$, where $\lambda_{\text {bulk }}$ is annihilation rate for free positrons annihilating in the bulk, i.e., free of defects region, $D_{+}$is the bulk positron diffusion coefficient $k_{0}$ is trapping rate parameter which is proportional to the defect concentration $C_{V}: k_{0}=\mu C_{V}$. The defect is an open volume defects which can trap positrons with a specific trapping rate denoted as $\mu$. In relation (1) it is assumed additionally that all positrons which reach the surface are trapped and annihilate there. Function $p(x, E)$ represents positron implantation profile, usually given by Makhovian function as follows:

$$
p(x, E)=\frac{m x^{m-1} x_{0}^{m}}{\exp }\left(-\left(\frac{x}{x_{0}}\right)^{m}\right),
$$

where 


$$
x_{0}=\frac{A_{1 / 2}}{\rho(\ln 2)^{1 / m}} E^{n} .
$$

$\rho$ represents the density of the implanted medium and $n$, $m$ and $A_{1 / 2}$ are parameters determined from the Monte Carlo simulation, e.g., within the GEANT 4 code. For silver they are as follows: $A_{1 / 2}=3.27 \mu \mathrm{g} \mathrm{cm}^{-2} \mathrm{keV}^{-n}$, $n=1.576, m=1.636$, see Ref. [21]. The mean positron implantation depth can be expressed as follows:

$$
\bar{x}=\Gamma(1+1 / m) x_{0},
$$

where $\Gamma$ is gamma function.

\section{References}

[1] P. Hosemann, Rev. Accl. Sci. Tech. 04, 161 (2011).

[2] W. Wesch, E. Wendler, Ion Beam Modification of Solids: Ion-Solid Interaction and Radiation Damage, Springer, 2016.

[3] G.S. Was, Fundamentals of Radiation Materials Science: Metals and Alloys, 2nd ed., Springer, Berlin 2016.

[4] R.W. Hamm, M.E. Hamm, Industrial Accelerators and Their Applications, World Sci., 2012.

[5] S. Klaumunzer, G. Schumacher, Phys. Rev. Lett. 51, 1987 (1983).

[6] Z. Wang, Y. Jin, M. Hou, G. Jin, Nucl. Instrum. Methods Phys. Res. B 169, 98 (2000).

[7] Yu.P. Sharkeev, B.P. Gritsenko, A.V. Fortuna, A.J. Perry, Vaccum 52, 247 (1999).

[8] Yu.P. Sharkeev, E.V. Kozlova, Surf. Coat. Technol. 158-159, 219 (2002).
[9] N. Didenko, E.V. Kozlov, Yu.P. Sharkeev, A.S. Tailashev, A.I. Rjabchikov, L. Pranjavichus, L. Augulis, Surf. Coat. Technol. 56, 97 (1993).

[10] Chenyang Lu, Ke Jin, L.K. Béland, Feifei Zhang, Taini Yang, Liang Qiao, Yanwen Zhang, Hongbin Bei, H.M. Christen, R.E. Stoller, Lumin Wang, Sci. Rep. 6, 19994 (2016).

[11] P. Mazzoldi, G. Mattei, L. Ravelli, W. Egger, S. Mariazzi, R.S. Brusa, J. Phys. D Appl. Phys. 42, 115418 (2009).

[12] A. Kinomura, R. Suzuki, T. Ohdaira, N. Oshima, K. Ito, Y. Kobayashi, T. Iwai, J. Phys. Conf. Series 262, 012029 (2011).

[13] R.J.M. Campillo, E. Ogando, F.J. Plazaola, J. Phys. Condens. Matter. 19, 176222 (2007).

[14] J. Kansy, Nucl. Instrum. Methods Phys. Res. A 374, 235 (1996).

[15] J.F. Ziegler, The Stopping and Range of Ions in Solids, Pergamon, New York 2003

[16] P. Horodek, J. Dryzek, V.A. Skuratov, Radiat. Phys. Chem. 122, 60 (2016).

[17] J. Dryzek, M. Wróbel, Tribol. Lett. 55, 413 (2014).

[18] J. Dryzek, Appl. Phys. A 114, 465 (2014).

[19] P. Horodek, J. Dryzek, V.A. Skuratov, Vacuum 138 , 15 (2017)

[20] J. Dryzek, Nucl. Instrum. Methods Phys. Res. B 196 186 (2002).

[21] J. Dryzek, P. Horodek, Nucl. Instrum. Methods Phys. Res. B 266, 4000 (2008). 